\title{
INFLUENCE OF DESIGN FEATURES ON THE STRUCTURAL INTEGRITY OF THREADED PIPE CONNECTIONS
}

\author{
T. Galle ${ }^{1}$, W. De Waele ${ }^{1}$, P. De Baets ${ }^{1}$, J. Van Wittenberghe ${ }^{1}$ \\ ${ }^{1}$ Ghent University, Laboratory Soete, Belgium
}

\begin{abstract}
When drilling and completing High Pressure High Temperature (HPHT) wells, the applied casing and tubing have to be able to withstand extreme conditions. Very often, the threaded connections are the most critical components in the entire chain. The overall design of several threaded and coupled (T\&C) connections (eg. VAM TOP, JFEBear and the newest PatriotTC) is very similar. However, even by modifying one single feature of the geometry, an improved structural integrity (eg. fatigue resistance, leak tightness or collapse strength) can be obtained. This paper gives an overview of the most important geometrical features and discusses their alleged effects on the structural integrity of the coupling.

At first, different thread designs were compared with each other and the effects on maximum allowable torque during make-up and tension during service conditions were evaluated. Secondly, literature shows that the use of an adequate torque shoulder is favourable, especially during the make-up stage. When designing connections for purposes involving gasses, a gastight seal is required. The third part of this paper discusses the influences on the sealability and performance limits when making changes to the sealing area. At last, the effect of overall changes (such as stress relief grooves) in the design and their consequences during running is shortly discussed. Based on this review, design guidelines for 'optimized' premium T\&C couplings can be formulated.
\end{abstract}

Keywords Design, Threaded Connection, Pipeline

\section{INTRODUCTION}

When drilling and operating oil and gas wells, the use of threaded pipe connections is favourable because of the inherent ability to demount and reuse the applied drill strings, tubing and casings. By applying such couplings, the overall cost of drilling and maintaining wells is reduced [1].

In recent years, the traditional OCTG (Oil Country Tubular Goods) products are unable to meet the desired requirements because of the exposure to the harsh conditions of High Pressure High Temperature (HTHP) wells. Due to the increasing development of these wells to reach remote gas and oil reserves, the enhancement of oil and gas tubular threaded joints is a growing economy with an increasing amount of players. Semi-premium and premium threaded connections are designed to fulfil the additional needs of improved reliability, leak resistance and performance. Figure 1 gives a brief overview of the evolution in the field of premium connections. In 1967, the depth of oil and gas wells had increased to over $4500 \mathrm{~m}$ and the development [2] and research [3] of threaded couplings has increased significantly.

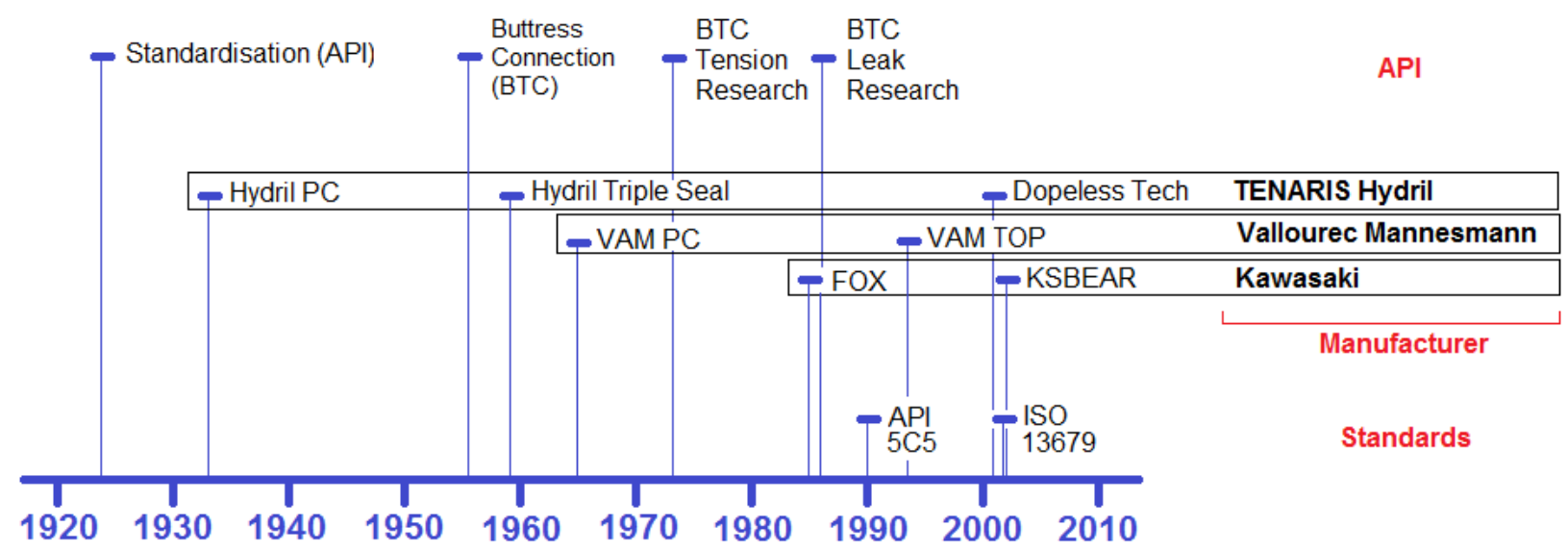

Figure 1: Timeline - evolution of premium connections and the three leading manufacturers with some of their most important T\&C connections [4] 
In the design of new premium connections (PC in Figure 1), three development phases have to be considered. During the first phase, the basic geometrical concept of a connection with chosen nominal size and material grade is engineered to withstand higher loads. Once the final basic geometry is established, the design is extrapolated to a variety of sizes and grades to create a new family of connections. Finally, in the third phase, the newly designed connections are tested in accordance with existing standards and procedures such as described in API 5C5 or ISO 13679. When these tests are successful, the connection should pass additional field-tests and is then considered fit for duty.

Possible adjustments of thread, torque shoulder and sealing area to enhance the design are considered in this paper. A discussion on improvement of fatigue life is reported in a companion paper [5].

\section{TYPES OF THREADED PIPE CONNECTIONS}

Originally, a sealed threaded pipe joint based on API buttress connectors (API BTC in Figure 1) patented by Samuel Webb [6] provided excellent joint strength. Mismatched leads closed the thread clearance on the stab flanks and provided the required leak resistance as can be seen in Figure 2. Due to improved manufacturing techniques, tighter tolerances are being held and clearances between the stab flanks are achieved, lowering leak resistance [7]. As a result, two new types of connections with an additional seal are developed to ensure the required leak resistance. One group consists of connections with a metal-to-metal seal providing gas tightness, named premium connections. The other group, without a metal-to-metal seal and water type sealability, is called semi-premium connections.

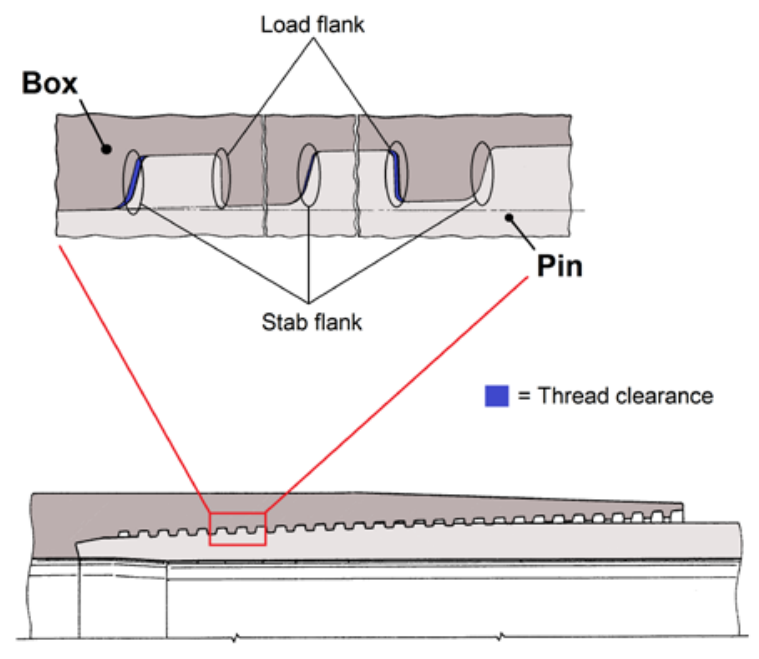

Figure 2: API Buttress thread with a mismatched lead reduces thread clearance resulting in excellent joint strength [6]

A

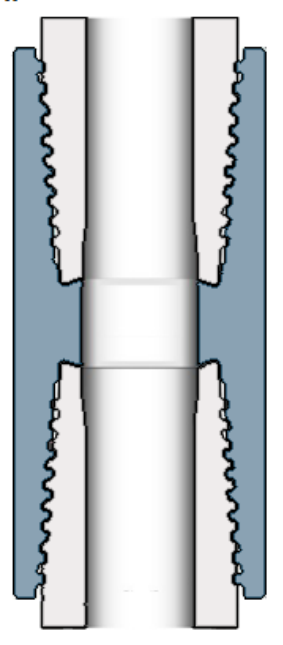

B-1.

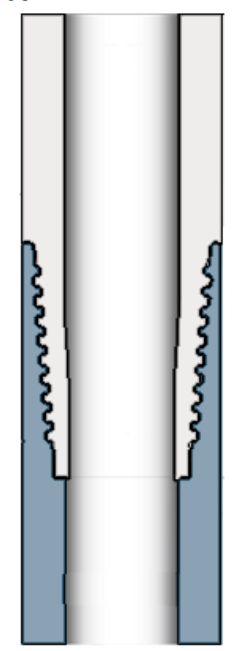

B-2.

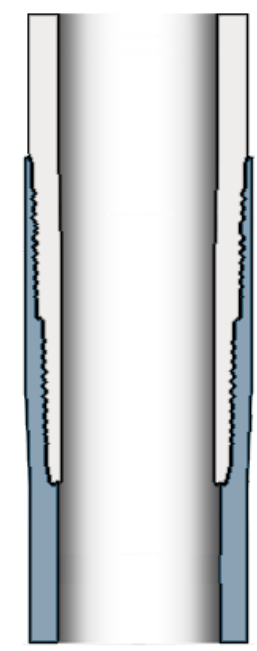

B-3.

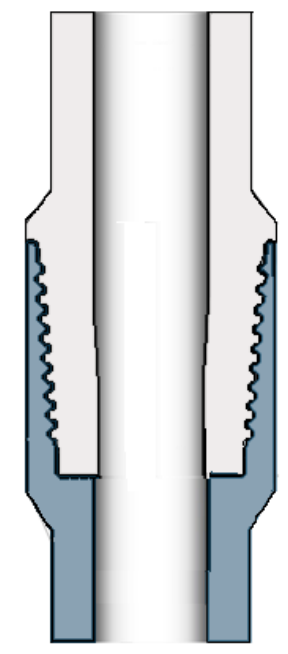

Figure 3: Different types of threaded connections with:

A. threaded and coupled connection

B. Integral connections: 1. integral flush, 2. integral semi-flush, 3. Integral upset [8] 
Figure 3 shows different types of premium threaded connections which are currently commercially available. Out of these types, two major groups can be defined. At one hand, the Threaded and Coupled (T\&C) connections and at the other hand the integral connections, which do not require special coupling stock.

$T \& C$ connections are less sensitive to stress concentrations and have proven to have higher tension ratings in comparison with integral connections. Even ratings exceeding the pipe body can be achieved. This implies that the strength of the connection in tension is equal or greater than the pipe body tensile yield strength. However, special attention is required when torsion or external pressure is applied. High leak resistance is guaranteed on static surfaces due to the variety of seal configurations which are possible.

The second group are the integral connections. With a sufficiently thick wall, these connections are efficient if external pressure is applied. When the outer diameter of the connection does not exceed the outer diameter of the pin, the connection is called an integral flush connection. In case the connection's outer diameters differs slightly from the pipe body, the connection is known as a semi-flush connection. To neutralize the reduced connection's joint strength due to the reduced wall thickness at the threaded area, a cold working process called swaging of the pin and/or box can be applied. AB Bradley et al [9] indicated that when no thermal relief is applied, a loss of sour service performance is obtained. A last member of the integral connections family is the upset integral connection, a type which is often used in tubing and drill strings. This expensive connection is limited by inner diameter and wall thickness.

It is worth noting that weld-on connectors (upset-type connections) are an additional type of threaded connection. However, because no threads are machined on the pipe body, they are considered to be connectors for pipe and not pipe connections.

\section{GEOMETRICAL DETAILS}

As stated before, the geometry of a premium connection dictates its success in the field. Figure 4 shows an API buttress threaded connection during make-up (A), with additional tensile loading (B) and with additional tension and internal pressure (C). The goal of the designer is to reduce plastic deformation and stress concentrations throughout the connection to increase its performance limits.

A.

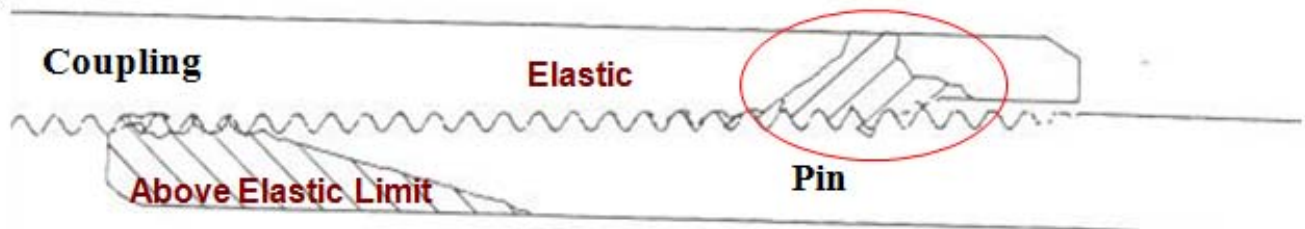

B.

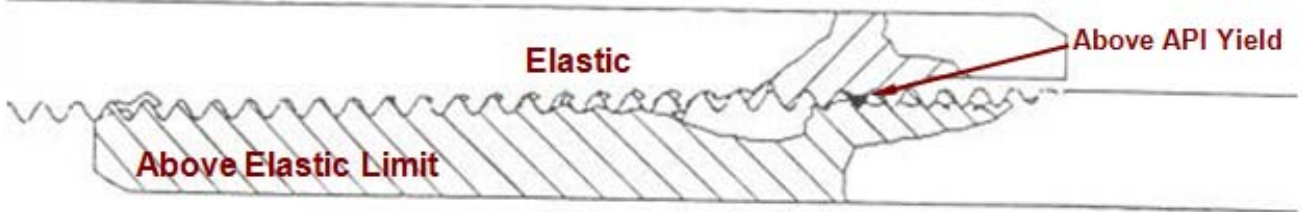

C.

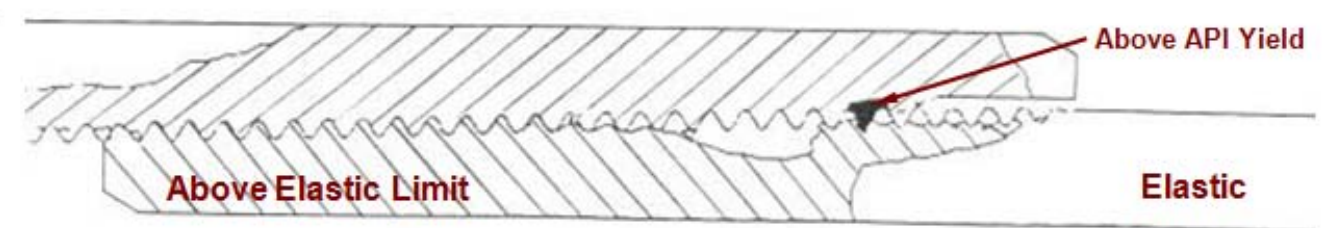

Figure 4: Yielding at working loads with:

A. Make-up, B. Make-up with tension, C. Make up with tension and internal pressure [4]

Most premium connections show comparable design features and have three major geometrical characteristics which are frequently altered to improve performance. The areas of interest are the thread, torque shoulder and metal-to-metal seal which are visible in Figure 5. 


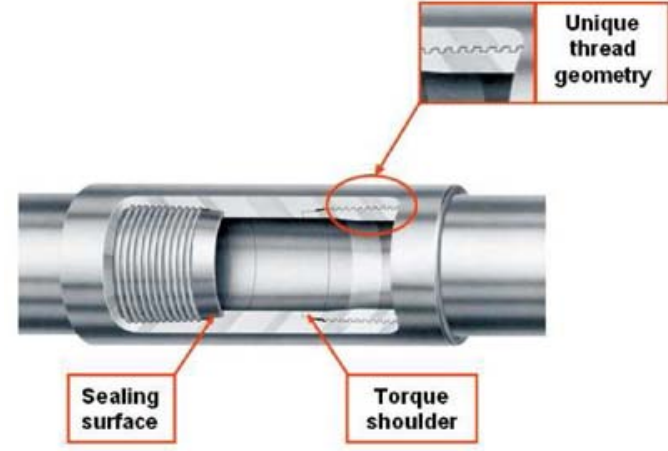

Figure 5: Overall view of a premium threaded connection with indication of the three major design features [8]

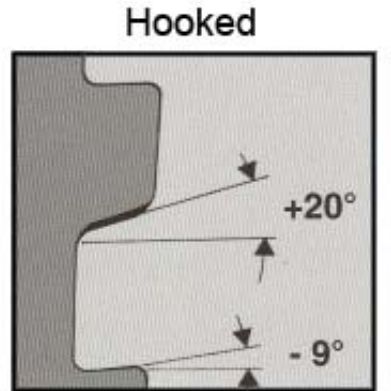

API Buttress

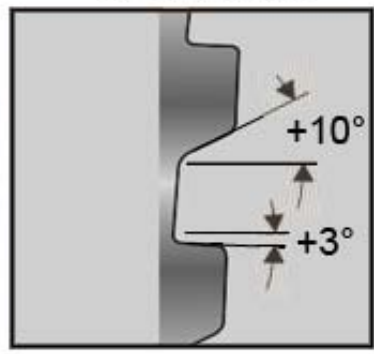

Figure 6: A hooked thread profile in comparison with the regular API buttress [8]

\subsection{Thread design}

Because of the presence of a metal-to-metal seal, the threads of premium connections are not required to interact as a pressure seal and can therefore be designed to maximize structural integrity. For this reason, API buttress type thread or a modified hooked thread with a negative load angle are commonly used (Figure 6). Despite the difficulties of machining a reversed load flank, the hooked thread design often dominates over a common API buttress profile. When tension is applied, the negative oriented load flank in combination with a positive stab flank, also known as a dovetail, reduces outward forces and eliminates radial separation between pin and box, resulting in a connection which is immune to jump-out failures and has higher tensile loading limits. In compression, most of the forces are supported by the stab flank and the hooked thread profile offers no significant advantages in comparison with the conventional buttress thread.

In a conventional thread design, an external tension load is mainly carried by the last engaged thread (LET) of the box. To realise a more uniform and favourable the stress distribution over the threaded surface, K.Yamamoto et al [10] suggested a pitch change concept as illustrated in Figure 7.

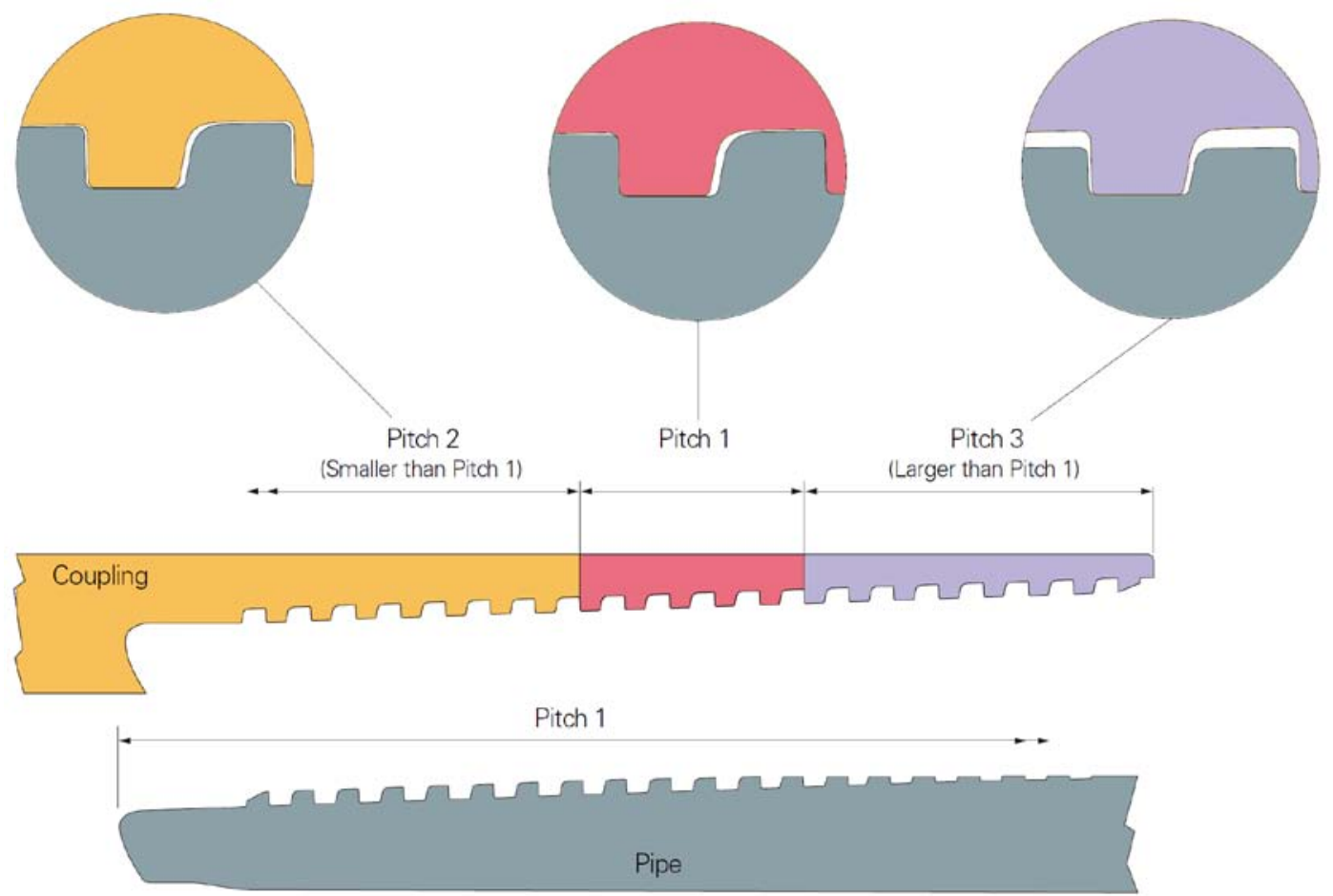

Figure 7: The pitch-change concept.

At pitch 2, the gap between the load flanks closes when made up. The standard pitch 1 concentrates the loads in the centre and the gap between the load flanks of the third pitch closes when axial tension is applied. (FOX connection, JFE) [11]

Figure 8 indicates that this concept improves the contact pressure distribution with reduced peaks at the end, resulting in better anti-galling properties and joint strength. 


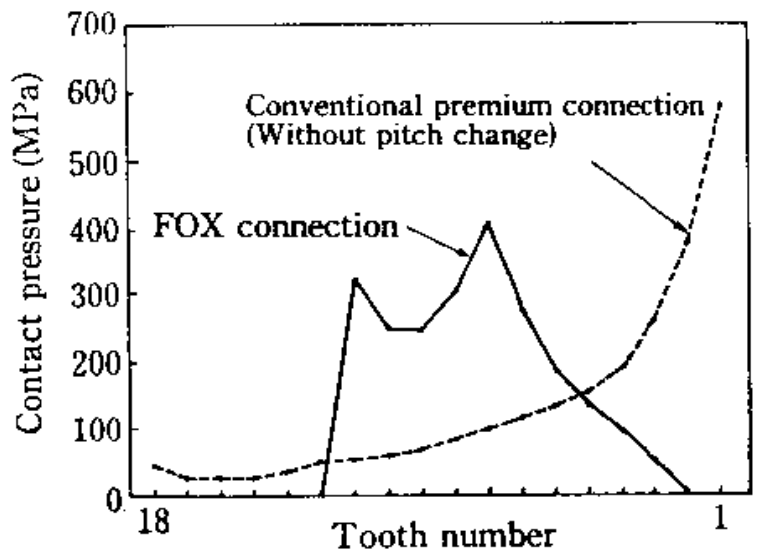

Figure 8: The pitch-change concept (FOX connection, JFE).

Peak pressures are reduced and pressure-distribution is enhanced [10]

A tapered thread has the inherent ability to establish a certain make-up torque on the thread itself. The steeper the taper, the higher the resistance to cross threading (Figure 9), also known as crest to crest engagement, but the thinner the applicable pin nose (see further). In order to increase resistance to cross threading without reducing the number of threads per inch and maximizing the pin thickness, it is possible to apply a tapered crest and root parallel to the pipe axis [12]. Figure 10 shows that the wider clearance (perpendicular to the connection axis) between thread root and crest allows greater self-alignment, resulting in easier stabbing during make up. This way, the running ability of the threaded connection is increased.

When a chamfer is added to the stabbing flank of the box, the risk of galling due to stabbing damage is further reduced.

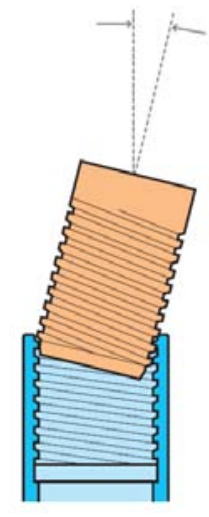

Figure 9: Cross threading due to misalignment during assembly [8]

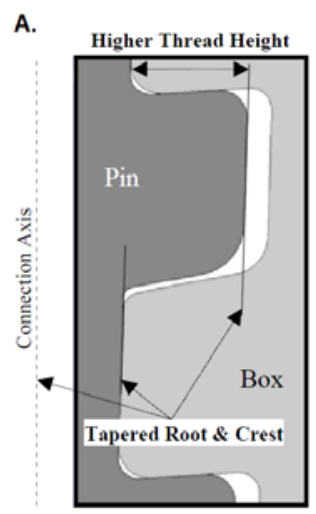

B.

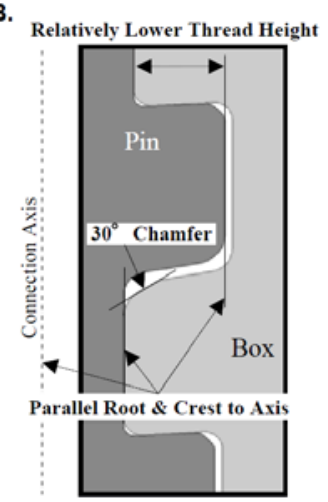

Figure 10: Crest/root taper parallel to the pipe body axis maximizes the running ability A. basic design, B. VAM21 [12]

\subsection{Shoulder design}

As described above, applying a certain make-up torque is possible by adding a taper to the threaded area. However, this method does not give the ability to apply the high torques required in HTHP wells. To such cases, the use of a shoulder is required. A shoulder can be applied in two different ways, either by using a pin to pin contact (Figure 11) or by adding a torque shoulder to the connection itself (Figure 12).

The pin nose of one pin can be used as the torque shoulder of the other pin. This allows reducing the outer diameter of the coupling, resulting in a reduced minimum required clearance. However, using a shoulder designed in the coupling is useful as a positioning feature for easy make-up and gives the opportunity to control the applied torque. The shoulder can transfer stored torque energy to a nearby seal to maintain contact pressure, resulting in favourable seal properties. It has to be pointed out that an increase in contact pressure increases the risk of galling significantly.

A connection equipped with a reverse torque angle, as illustrated in Figure 13, causes a wedge effect. This phenomenon gives the connection self-alignment [14], radial stability and improved structural strength, which is magnified by the inverse angle of the shoulder itself.

Generally, the larger the contact surface of the shoulder, the higher the torque capabilities. 


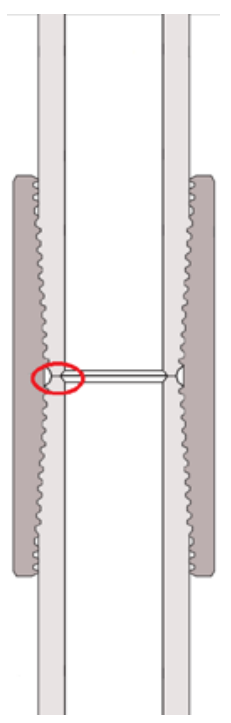

Figure 11: Pin-to-pin contact

(Seal-Lock HT, Hunting) [13]

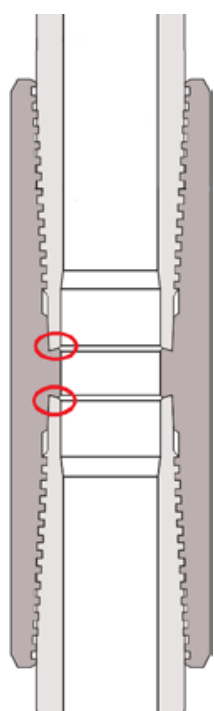

Figure 12: Box with torque shoulder

(Seal-lock APEX, Hunting) [13]

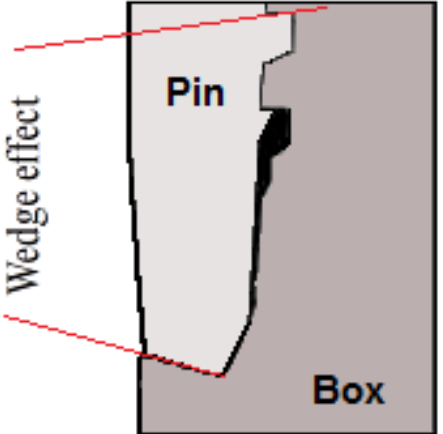

Figure 13: The wedge effect determined by the angle of the torque shoulder and the load flank of the thread increases the self-aligning abilities of the connection (VAM TOP) [8]

\subsection{Sealing area design}

In contrast with semi-premium connections, premium connections do not use a thread seal but an extra sealing feature is added to ensure pressure integrity. Usually, a metal-to-metal seal is located near the torque shoulder to energize the sealing surface. Simulations [10] have shown that using the thread-changeconcept can increase the contact surface energy of the seal as well.

Unlike polymer seals such as teflon rings, a metal-to-metal seal is one of the most reliable seals and is able to provide a gastight seal under high pressure and extreme temperatures. As a result of this surface contact, special attention has to be given to the surface finish (usually characterized by Ra between 0.8 and $3.2 \mu \mathrm{m} \mathrm{[15])} \mathrm{and} \mathrm{the} \mathrm{risk} \mathrm{of} \mathrm{galling} \mathrm{has} \mathrm{to} \mathrm{be} \mathrm{taken} \mathrm{into} \mathrm{account} \mathrm{when} \mathrm{designing} \mathrm{the} \mathrm{sealing} \mathrm{area.} \mathrm{The}$ commonly used configurations are a cone-to-cone sealing (a tapered surface on the pin and box) or a sphere-to-cone sealing (a toroidal surface on the pin and a tapered surface on the box). The steeper the taper, the higher the pressure integrity and the higher the risk of galling.

Figure 14 gives an example of the behaviour of these two variants. The cone-to-cone configuration gives a higher maximum contact pressure (and risk of galling), but has a limited seal length in comparison with the sphere-to-cone design. From these results, it is not possible to determine which design has the best sealing capacity and additional experiments have to be implemented [16].

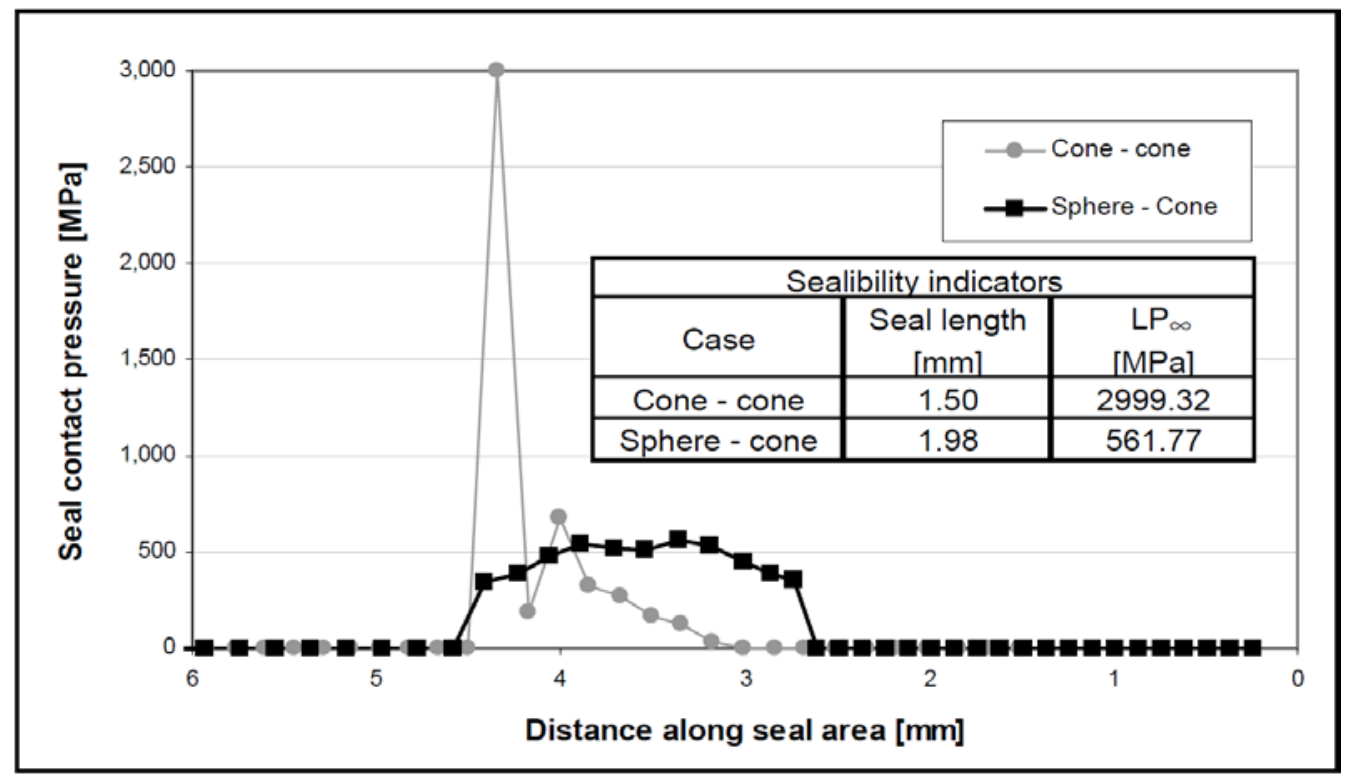

Figure 14: Comparison of a cone-to-cone and a sphere-to-cone seal design.

The sphere-cone configuration reduces peak pressures and improves pressure-distribution. [16] 
K. Yamamoto et al [10] designed a triple radii seal with three blended arcs. The use of different radii triggers a reduction of stress concentrations which leads to an increased galling and overtorque resistance resulting in a high performance seal. Due to the complex shape of this seal, repair is not possible without affecting the seal integrity [17].

Despite their excellent performance, metal-to-metal seals are vulnerable and any kind of distortion will lead to leaking. For this reason, the design has to be optimized to reduce the ability of inflicting damage during handling and make-up. One way of protecting the surface is to design the taper guide as given in Figure 15.

A.

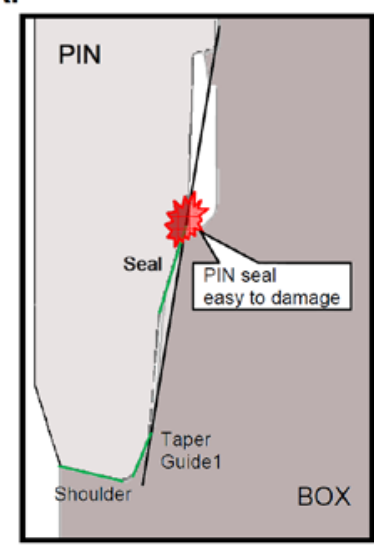

B.

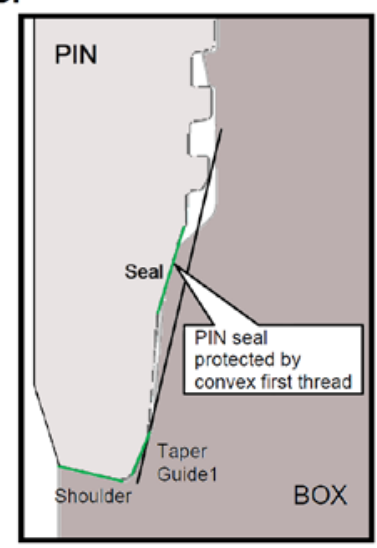

Figure 15: Seal protection mechanism with: A. a conventional premium connection, B. VAM21 [12]

The use of an internal and external seal in a design can be beneficial. When an internal seal protects the thread against internal pressure and the external seal against external pressure, the risk of pressure migration through the thread helix and thus the risk of leaking is reduced considerably.

\subsection{Various improvements}

The design of a threaded coupling is not limited to the three features discussed higher. In order to improve performance with the same or a reduced amount of material, small but significant adjustments can be made.

When connecting pipes with threaded connections, compound greases with graphite are used to reduce the risk of galling and to provide leak tightness in semi-premium connections [18]. Despite the necessity of this lubricant, the grease can initiate a pressure build-up in the threads during make-up and can cause the connection to fail. For this reason, pressure relief grooves can be manufactured in the connection as shown in Figure 16. During make-up, the excess of grease is transferred to this chamber and a pressure build-up is avoided. As from 2003, the disadvantages of lubricants can be avoided by using dopeless technology [19]. This technology consists of a surface treatment instead of greases to avoid galling. The surface treatment of these dry connections often consists of the use of a soft metal plating, a dispersion plating or a solid lubricant coating type [18].

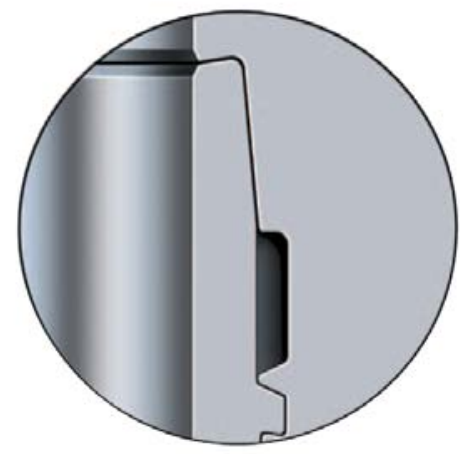

Figure 16: A lubricant relief groove avoids pressure buildup caused by grease. (Seal lock Apex, Hunting)

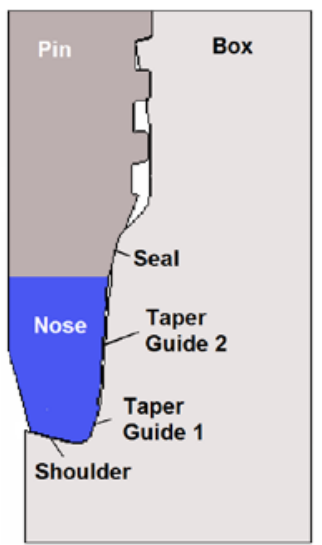

Figure 17: prolonging the pin with a nose increases stiffness (VAM21) [12]

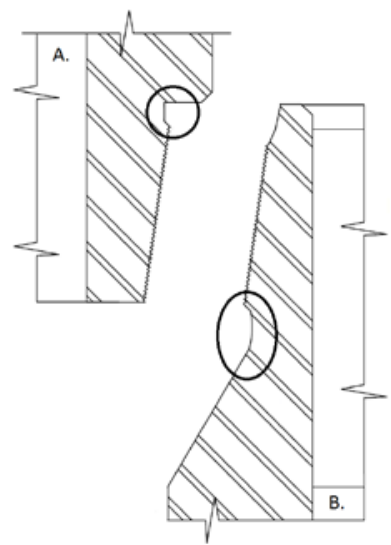

Figure 18: stress relief grooves at:

A. pin and B. box [22] 
The pin can be extended with a nose as illustrated in Figure 17. The thickness and length of the nose give extra stiffness to the configuration which is used to amplify the resulting forces of the negative torque shoulder and enhances pressure integrity [20]. At the same time, this nose can be used to protect the seal contact surface against handling and prevents eccentric contact by adding a guiding surface which is used for positioning the box and the pin during stabbing.

When couplings are designed for drill pipes, fatigue lifetime is a significant design requirement. To reduce stress concentrations at the Last Engaged Thread (LET), applying stress reduction grooves might prove beneficial [21]. Figure 18 shows the standard relief grooves for drill collars as described in API 7 [22].

\section{CONCLUSIONS}

During the design process, special attention is given to several aspects of the threaded connections. By altering the thread, usually a buttress or hooked profile, structural integrity against axial forces, pressure and bending is provided. The design of the shoulder determines the maximal amount of pretension that can be applied and the seal is necessary to prevent the connection from leaking due to internal and/or external gas pressure. It is important to realize that the design is not limited to these three parts of the connection. Other small geometrical enhancements, choice of materials and surface finish can be used to finalize a new connection which is able to withstand harsher conditions and greater loads.

It is important to note that not only the mechanical behaviour of a connection is important. When put in service, all connections should be able to withstand (mis-)handling and vulnerable areas should be protected to ensure reliable performance.

At last, it should be pointed out that this paper describes trends and does not give any numerical results of geometrical changes. Further research is required to determine the effect of isolated changes and to quantify the resulting interactions of combined adaptations.

\section{REFERENCES}

[1] David Walters, Ricky Thethi, A Step Change Application of Threaded and Coupled Connections, 2H Offshore Inc., USA, 2002

[2] P. D. Weiner, Martin E. True, A Method of Obtaining Leakproof API Threaded Connections In Highpressure Gas Service, Drilling and Production Practice, 1969, American Petroleum Institute

[3] API, WI 2317: Tech Report on LTC/BTC Performance Properties and Leak Resistance: THREAD COMPOUNDS, 2006

[4] API, Presentation: Performance properties: requirements for leak resistance of API connections (LTC/BTC), http://mycommittees.api.org/, December 2010

[5] J. Van Wittenberghe, P. De Baets, W. De Waele, T. Galle, T.T. Bui and G. De Roeck, Design characteristics that improve the fatigue life of threaded pipe connections, Sustainable Construction and Design (SCAD), Ghent, Belgium, 2011

[6] Patent US2772102 - Samuel Webb, Pleasant Hills, Pa. Sealed Threaded Pipe Joint. Patent dated Nov. 27, 1956. Dedication filed Apr. 5, 1962, by the assignee, United Stated Steel Corporation.

[7] API REPORT 86-53 Document Information, Investigation of Leak Resistance of API Buttress Connector, American Petroleum Institute, 1987

[8] Vallourec \& Mannesmann, VAM book, http://www.vamservices.com, December 2010.

[9] A. B. Bradley, Premium Connection Design, Testing, and Installation for HPHT Sour Wells, Society of Petroleum Engineers Inc, SPE High Pressure-High Temperature Sour Well Design Applied Technology Workshop, 17-19 May 2005, The Woodlands, Texas, U.S.A., SPE 97585

[10] K. Yamamoto, K. Kobayashi et al, Stress Analysis of Premium Threaded Connection "FOX" by Finite Element Method, Kawasaki Steel Giho, 20 (1989) 3, pp. 202-207

[11] JFE Steel Corporation, FOX Premium Threaded Connection

[12] M. Sugino, K. Nakamura and S. Yamaguchi, Development of an Innovative High-Performance Premium Threaded Connection for OCTG, Offshore Technology Conference, 3-6 May 2010, Houston, Texas, USA, OTC 20734

[13] World Oil, 2008-09 Casing Reference Tables, November 2008 
[14] Patent WO/2010/122431 - Tenaris, Threaded Joint for Tubes, Pipes and the Like, Patent dated October 28, 2010. Filed April 22, 2010

[15] Couplings, http://www.exprobase.com/Default.aspx?page=696, December 2008

[16] Eduardo N. Dvorkin, Rita G. Toscano, Finite element models in the steel industry: Part II: Analyses of tubular products performance, Computers \& Structures, Volume 81, Issues 8-11, K.J Bathe 60th Anniversary Issue, May 2003, Pages 575-594

[17] Hunting, Recommendations for the repair \& re-use of FOX premium threaded connections, 1994

[18]E. Tsuru, Y. Tsukano et al, Dope-free Type Premium Connection for Oil Country Tubular Goods, Nippon Steel Technical Report No. 81, January 2000

[19] Tenaris Hydril, Dopeless Technology, brochure, version 01, October 2010

[20]A. Muradov, J. E. Smith, Development of External Pressure Resistant Tool Joint for Under-balanced Drilling, SPE/IADC Drilling Conference, Amsterdam, The Netherlands, 27 February - 1 March, 2001, SPE/IADC 68771

[21]R. Shilling, M.L. Payne, High-Strength Treaded and Coupled Connectors for Dynamic Riser Applications, International Oil Conference and Exhibition, Veracruz, Mexico, 27 - 30 June, 2007, SPE 108708

[22] API Specification 7, Specification for Rotary Drill Stem Elements, American Petroleum Institute, thirtyninth edition, December 1998. 